\title{
Nuclear Reaction Studies with Exotic Boron Beams
}

\author{
V. Guimarães \\ Instituto de Física, Universidade de São Paulo, C.P. 66318, 05389-970, São Paulo, SP, Brazil
}

Received on 20 October, 2003

\begin{abstract}
Spectroscopic investigation, on exotic nuclei, performed in two different experiments with secondary radioactive boron beams are discussed. Spectroscopic factors for the ${ }^{13} \mathrm{~B}+\mathrm{n}$ bound-states are obtained by measuring momentum distribution of the ${ }^{13} \mathrm{~B}$ residual nucleus from knockout reaction of the ${ }^{14} \mathrm{~B}$ beam at intermediate energy. The momentum distributions are measured in coincidence with the emited $\gamma$-rays from the excited residual ${ }^{13} \mathrm{~B}$ nucleus allowing spin-parity assignment and partial cross section determination. In the other experiment, a deuteron transfer, ${ }^{2} \mathrm{H}\left({ }^{8} \mathrm{~B}, \alpha\right){ }^{6} \mathrm{Be}$, reaction with low energy ${ }^{8} \mathrm{~B}$ beam are used to search for resonances in the unbound ${ }^{6}$ Be nucleus. The ANC - Asymptotic Normalization Coefficient method are presented and astrophysical implications of these two experiments are discussed.
\end{abstract}

\section{Introduction}

Spectroscopic studies of short-lived radioactive nuclei far from the valley of beta stability is one of the major active field in nuclear physics in nowadays. These exotic nuclei are weakly bound systems and some of them have been shown "halo" and "skin" properties due to a very diffuse surface. To performe nuclear spectroscopic investigation on such nuclei direct reaction processes that add or remove one or few nucleons, as direct stripping and pickup reactions, and from which one can identify single-particle orbitals, energies and their occupancies are commonly used. Recently, reactions with radioactive beams have been proved to be also very powerfull tools to performe spectroscopic investigation on exotic nuclei, allowing even to probe resonances in unboud nuclei [1]. At NSCL-Michigan State University, another technique has been developed for spectroscopic studies of rare and exotic nuclei. The technique consists of measuring partial cross section and longitudinal momentum distributions corresponding to specific final states of the fragments produced from a breakup reaction at intermediate enegies. These measurement and the model used to interpret the data, which combines spectroscopic factors derived from the shell model with single-particle cross sections calculated in a eikonal model, can account for the intrinsic structure and the reaction mechanism [2-5].

There is a strong indication that the dissociation reaction of valence nucleon in a loosely bound nuclei is peripheral and dominated by nuclear surface [3, 4]. Thus, spectroscopic factors, obtained from dissociation of the valence neutron in ${ }^{14} \mathrm{~B}$, can be used to draw inferences about the inverse ${ }^{13} \mathrm{~B}$-neutron capture reaction by obtaining the ANC (Asymptotic Normalization Coefficients) of single-particle wave functions, which can be of astrophysical interest.

Also, information on energy and width of states and resonances in light mass exotic nuclei is of strong relevance for astrophysics. For instance, a possible resonance in low energy part of the ${ }^{3} \mathrm{He}\left({ }^{3} \mathrm{He}, 2 \mathrm{p}\right){ }^{4} \mathrm{He}$ reaction spectrum [6] would correspond to a level at about $11 \mathrm{MeV}$ excitation energy in the ${ }^{6} \mathrm{Be}$ nucleus. Althgough a narrow resonance is not expected in this unbound nucleus, the possible existence of broad resonance is not ruled out [7]. Such resonance in ${ }^{6} \mathrm{Be}$, which has not been observed yet, would favor the ${ }^{3} \mathrm{He}\left({ }^{3} \mathrm{He}, 2 \mathrm{p}\right){ }^{4} \mathrm{He}$ reaction in detriment to the other ${ }^{3} \mathrm{He}\left({ }^{4} \mathrm{He}, \gamma\right){ }^{7} \mathrm{Be}$ reaction in the pp-chain in the Sun; and thus, it would have some direct implication in the neutrino solar problem.

In this paper we present the results of spectroscopic investigation on exotic nuclei performed in two different experiments with radioactive boron beams. One is an experiment on breakup reaction using intermediate energy ${ }^{14} \mathrm{~B}$ beam to obtain spectroscopic factors for the ${ }^{13} \mathrm{~B}+\mathrm{n}$ boundstate and the other is an experiment on transfer reaction using low energy ${ }^{8} \mathrm{~B}$ beam to search for resonances in the unbound ${ }^{6}$ Be nucleus.

\section{Breakup reaction with intermediate energy ${ }^{14} \mathbf{B}$ beam}

Using neutron-rich radioative ${ }^{14} \mathrm{~B}$ beam at $60 \mathrm{MeV} /$ nucleon obtained from the A1200 separator at NSCL - Michigan State University we investigated the knockout reaction ${ }^{9} \mathrm{Be}\left({ }^{14} \mathrm{~B},{ }^{13} \mathrm{~B}+\gamma\right) \mathrm{X}$. The secondary ${ }^{14} \mathrm{~B}$ radioactive secondary beam with $59.2 \pm 0.3 \mathrm{MeV}$ per nucleon, was produced and selected by the A1200 fragment separator at NSCL [8]. The ${ }^{14} \mathrm{~B}$ beam was then transmitted to a target chamber where a neutron was removed during an interaction with the Be target, leaving the ${ }^{13} \mathrm{~B}$ core in its ground state or in an excited state. The $\gamma$-rays from the decay of ${ }^{13} \mathrm{~B}$ excited states were detected by an array of 38 cylindrical $\mathrm{NaI}$ (Tl) detectors [9] which completely surrounded the target. Recoiling ${ }^{13} \mathrm{~B}$ core fragments were momentum-analyzed using the S800 spectrograph [10], where the momentum distributions of ${ }^{13} \mathrm{~B}$ fragments corresponding to removal of single 
TABLE 1.Partial cross sections in mb for each of the final states populated in ${ }^{13} \mathrm{~B}$. The theoretical value for the cross section is the sum of the two components multiplied by the corresponding spectroscopic factor and kinematic center of mass correction. The experimental value, the reduction factor and the extracted ANC is given in the last three columns.

\begin{tabular}{cccccccccc}
\hline $\mathrm{E}(\mathrm{Mev})$ & $\mathrm{J}^{\pi}$ & $l$ & $C^{2} S_{s p}$ & $\sigma_{\text {strip }}$ & $\sigma_{\text {diff }}$ & $\sigma_{\text {theo }}$ & $\sigma_{\text {exp }}$ & $R_{S}$ & $A N C_{l}$ \\
\hline 0.00 & $\frac{3}{2}^{-}$ & 0 & 0.662 & 82.4 & 53.9 & 98.8 & $113(15)^{a}$ & 1.14 & $0.73(10)$ \\
& & 2 & 0.306 & 35.0 & 16.5 & 17.0 & $14(3)^{a}$ & 0.82 & $3.9(9)$ \\
3.48 & $\frac{3}{2}^{+}$ & 1 & 0.407 & 24.1 & 9.7 & 14.8 & $18(3)$ & & \\
3.68 & $\frac{5}{2}^{+}$ & 1 & 0.886 & 23.5 & 9.4 & 31.4 & $30(5)$ & & \\
4.13 & $\frac{1}{2}^{-}$ & 2 & 0.0005 & 22.3 & 8.0 & 0.02 & $1.2(0.6)$ & & \\
\hline
\end{tabular}

(a) Cross section obtained by deconvolution of the $l=0$ and $l=2$ contributions to the longitudinal momentum distribution.

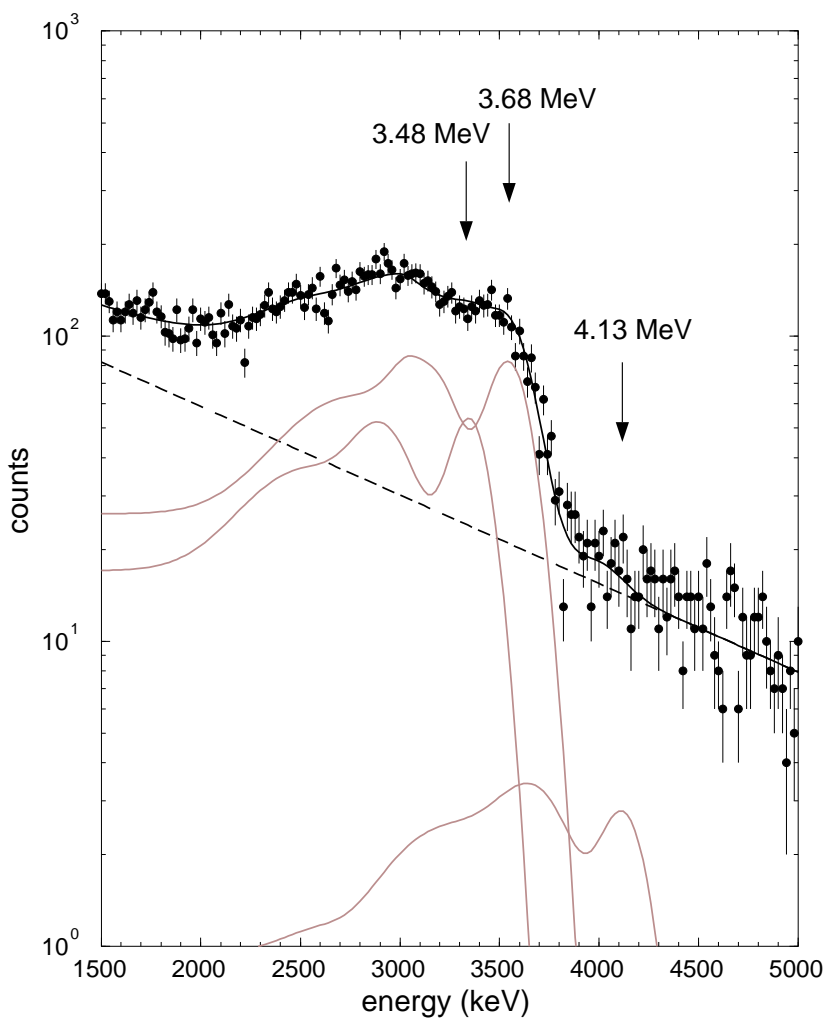

Figure 1. Doppler corrected energy spectrum of the $\gamma$-rays measured in coincidence with a ${ }^{13} \mathrm{~B}$ fragment in the NaI array. The solid line is the fit to the experimental data. The fit corresponds to an independent normalization of the simulated response functions for each individual $\gamma$-ray.

neutron from different orbitals in ${ }^{14} \mathrm{~B}$ were measured. The contribution from ${ }^{13} \mathrm{~B}$ core excitation was isolated by measuring the momentum distribution of excited ${ }^{13} \mathrm{~B}$ fragments in coincidence with $\gamma$-rays from their decay. The results on the momentum distribution of the ${ }^{13} \mathrm{~B}$ fragments and more details about this experiment can be found in ref. [5].

The energy spectrum of $\gamma$-rays in coincidence with ${ }^{13} \mathrm{~B}$ fragments is shown in Fig. 1. The solid curve is a fit to the data obtained via Monte-Carlo simulation using the code GEANT [11]. The simulation took into account Doppler broadening, the distortion of the shapes caused by the back transformation to the projectile rest frame, and the calculated $\gamma$-ray detection efficiencies. The background, primarily resulting from neutron reactions with the detector itself and the surrounding materials, was parameterized by a simple exponential dependence. Taking into account the 3.48 $\mathrm{MeV}, 3.68 \mathrm{MeV}$ and $4.13 \mathrm{MeV}$ transitions in ${ }^{13} \mathrm{~B}$, the fit to the experimental spectrum is excellent (the $\chi^{2}$ per degree of freedom is 1.08).

The ${ }^{13} \mathrm{~B}$ ground state has $\mathrm{J}^{\pi}=\frac{3}{2}^{-}$, resulting from a $\left[1 p_{3 / 2}\right]^{-1}$ proton configuration. Below $4.5 \mathrm{MeV}$, three negative parity and two positive parity excited states have been previously identified in ${ }^{13} \mathrm{~B}$ [12] but very little is known about their spins. Based on the $\gamma$-ray spectrum of Fig. 1 and by the fact that only the spectroscopic factors for oneneutron removal for the two first positive parity states and the first $\frac{1}{2}^{-}$state had significant spectroscopic strength [13], the spin assignments presented in Table I have been established [5]. Also the partial cross sections obtained from the absolute $\gamma$-ray intensities corrected by the computed efficiencies and the spectrometer acceptance are presented in Table I.

The single-particle cross sections for each final state of the core fragment were calculated by Tostevin [14] in a eikonal model assuming that there are two reaction mechanisms involved in the knock-out reaction: (i) nucleon stripping in which the halo nucleon interacts strongly with the target and leaves the beam, and (ii) diffraction dissociation in which the nucleon moves forward with essentially the beam velocity but away from the core. The theoretical cross sections for each states below 4.5 MeV, given in Table I, is given by the expression:

$$
\sigma_{t h e}=\left(\frac{A}{A-1}\right) \times C^{2} S \times\left(\sigma_{s t r}+\sigma_{d i f}+\sigma_{C}\right) .
$$

Here $\mathrm{A} /(\mathrm{A}-1)$ is a center-of-mass correction [16]. This factor is usually neglected but it can be important for a more precise comparison with data. Also the Coulomb contribution due to Be-target was calculated and it was found to be very small, $\sigma_{C}=2.3 \mathrm{mb}$ for the ground state. The best agreement with experiment is achieved with the spin assignments shown in Table I. Overall, it appears that the combination of the theoretical spectroscopic factors and computed singleparticle cross sections does a remarkable job of predicting the experimental yields. 


\section{The astrophysical S-factor and the ANC (Asymptotic Normalization Coefficient.)}

The capture cross section, $\sigma_{\mathrm{CAP}}$, for $A+x \rightarrow B+\gamma$ reaction can be calculated by the following equation [15]:

$$
\sigma_{\mathrm{CAP}}=\lambda\left|<I_{A x}^{B}(\mathbf{r})\right| \hat{O}(\mathbf{r})\left|\Psi_{i}^{(+)}(\mathbf{r})>\right|^{2},
$$

where $\lambda$ corresponds to a kinematic factor, $I_{A x}^{B}(\mathbf{r})$ is the radial overlap integral of the bound state $B=A+x, \hat{O}$ is the eletromagnetic transition operator and $\Psi_{i}^{(+)}(\mathbf{r})$ is the continuum single particle wave function.

For a peripheral capture the tail of the radial overlap integral is given asymptotically by correct Whittaker or Hankel function for a neutron capture,

$$
\begin{gathered}
I_{A x}^{B}=A N C_{A x} \times \frac{W_{-\eta, l+\frac{1}{2}}(2 \kappa r)}{r} \text { or } I_{A n}^{B} \\
=A N C_{A n} \times \frac{H_{l}(\kappa r)}{r}
\end{gathered}
$$

where $W_{-\eta, l+\frac{1}{2}}(2 \kappa r)$ is the Whittaker function, $H_{l}(\kappa r)$ is first order Hankel function, $\eta=Z_{a} Z_{x} e^{2} \mu / \kappa$ is the Sommerfeld parameter, $\kappa=\sqrt{2 \mu \epsilon} / \hbar, \mu$ and $\epsilon$ are the reduced mass and binding energy for the $A-x$ system, respectively. And, ANC is the Asymptotic Normalization Coefficient.

At low energies, the amplitude for the radiative capture cross section is dominated by contributions from large relative distances of the participating nuclei, and thus, by determining the asymptotic normalization coefficient (ANC) one can normalize the non-resonant part of capture reaction.

This normalization coefficient can be found from peripheral transfer reactions whose amplitudes contain the same overlap function as the amplitude of the corresponding capture reaction of interest. The transfer reaction as a way to obtain the ANC has been already tested and applied to obtain the S-factor $S_{17}(0)$ for the ${ }^{7} \mathrm{Be}(\mathrm{p}, \gamma)^{8} \mathrm{~B}$ capture, where the transfer reaction ${ }^{10} \mathrm{~B}\left({ }^{7} \mathrm{Be},{ }^{8} \mathrm{~B}\right){ }^{9} \mathrm{Be}$ was used [17]. In this reaction, the normalization coefficient for the ${ }^{9} \mathrm{Be}+\mathrm{p}={ }^{10} \mathrm{~B}$ vertex was known and the normalization coefficient for the ${ }^{7} \mathrm{Be}+\mathrm{p}={ }^{8} \mathrm{~B}$ was determined from the angular distribution and DWBA calculation. Imai et al. also applied this ANC method for neutron transfer reaction, ${ }^{12} \mathrm{C}(\mathrm{d}, \mathrm{p}){ }^{13} \mathrm{C}$ reaction [18]. The obtained $\mathrm{ANC}$ was used to normalize the non-resonant part of the ${ }^{12} \mathrm{C}(\mathrm{n}, \gamma){ }^{13} \mathrm{C}$ capture reaction. In a recent paper, Trache et al. [19] has suggested that the ANC can be extracted from one-nucleon breakup reaction of loosely bound nuclei.

Thus, for a knockout reaction the overlap function would to be given by:

$$
I_{A x}^{B}=\left(\frac{A}{A-1} \times C^{2} S \times R_{s}\right)^{2} \times R_{l}(r),
$$

where $R_{s}$ is a short range correction or reduction factor given by $R_{s}=\sigma_{\text {exp }} / \sigma_{\text {the }}$. This factor takes into account effects as short range of the nucleon-nucleon interaction, not considered in the spectroscopic factor $C^{2} S$ calculated in the single particle shell model.

By combining the equations [3] and [4] and using the definition of the reduction factor $R_{s}$ we can obtain the ANC from the breakup reaction at large distance $r_{L}$ as:

$$
A N C_{l}^{2}=\frac{\sigma_{e x p}}{\left(\sigma_{s t r}+\sigma_{d i f}+\sigma_{C}\right)} \times\left(\frac{r_{L} R_{l}\left(r_{L}\right)}{\text { Factor } \times H_{l}\left(\kappa r_{L}\right)}\right)^{2} .
$$

The ANC calculated for $\mathrm{n}+{ }^{13} \mathrm{~B}(\mathrm{gs})={ }^{14} \mathrm{~B}(\mathrm{gs})$ capture reaction corresponding to $2 s_{1 / 2}$ and $1 d_{5 / 2}$ components of the ${ }^{14} \mathrm{~B}$ ground state configuration is also presented in Table I. This coefficient is conveniently insensitive to specifications of nuclear-structure parameters since these structure details are factorized by the radial functions. The essential point here is that we can use experimental information from breakup reaction together with a eikonal reaction theory and shell model description to extract astrophysical informations in a similar way as the transfer reaction was used together with DWBA calculation for transfer reactions. With this coefficient one can calculate the $S$-factor for the ${ }^{13} B+n$ capture reaction correctly normalized.

\section{Transfer reaction with low energy ${ }^{8}$ B beam}

In a search for resonances in ${ }^{6} \mathrm{Be}$ we have measured the deuteron transfer reaction ${ }^{2} \mathrm{H}\left({ }^{8} \mathrm{~B}, \alpha\right){ }^{6} \mathrm{Be}$. The results of this experiment is also presented in ref. [20]. The experiment was performed at University of Notre Dame, USA. The secondary radioative ${ }^{8} \mathrm{~B}$ beam was produced using the ${ }^{3} \mathrm{He}\left({ }^{6} \mathrm{Li},{ }^{8} \mathrm{~B}\right)$ reaction. The ${ }^{8} \mathrm{~B}$ secondary beam was separated by the double superconducting-solenoid system ( Twinsol) [21]. The two superconducting solenoids work as thick lenses to collect and focus the secondary beam onto a $1.04 \mathrm{mg} / \mathrm{cm}^{2}$ thick $\mathrm{CD}_{2}$ secondary target. The laboratory energy of the ${ }^{8} \mathrm{~B}$ beam at the center of this target was 28.55 $\mathrm{MeV}$, with an overall energy resolution of $0.75 \mathrm{MeV}$ full width at half maximum (FWHM) and an average intensity of $1.0 \times 10^{3}$ particles per second. Ions with the same magnetic rigidity as the ${ }^{8} \mathrm{~B}$ beam were also present at the secondary target position, but they could be separated by using TOF signal. The TOF of the particles was obtained from the time difference between the E signal in a telescope and the RF timing pulse from the beam buncher. The particles produced by the reaction with ${ }^{8} \mathrm{~B}$ beam on the $\mathrm{CD}$ target were detected by four telescopes placed at $\theta_{\mathrm{LAB}}=10^{\circ}, 20^{\circ}, 30^{\circ}$ and $45^{\circ}$. Each telescope subtended a solid angle of about 12 msr. Also a carbon run was measured for the later target background subtraction. The ${ }^{6} \mathrm{Be}$ is unbound to ${ }^{4} \mathrm{He}+2 \mathrm{p}$ decay by $1.377 \mathrm{MeV}$ but the populated levels (resonances) in ${ }^{6} \mathrm{Be}$ by the ${ }^{2} \mathrm{H}\left({ }^{8} \mathrm{~B}, \alpha\right){ }^{6} \mathrm{Be}$ reaction would be observed as peaks in the detected $\alpha$ spectrum sitting on a background due at least in part to alternative breakup processes $\left({ }^{6} \mathrm{Be} \rightarrow\right.$ 
${ }^{4} \mathrm{He}+2 \mathrm{p}$ or $\left.{ }^{5} \mathrm{Li}+\mathrm{p}\right)$ corresponding to 3 or 4 -body phase-space background.

The only spectrum which shows some structure other than the ground state is the one measured at 45 degree. This spectrum can be seen in Fig. 2. Due to the low intensity of the ${ }^{8} \mathrm{~B}$ beams the statistics of this spectrum are very poor. However, as one can see, there is an evidence for a resonance at $11 \mathrm{MeV}$, which would correspond to a $9.6 \mathrm{MeV}$ in excitation energy of ${ }^{6} \mathrm{Be}$. This resonance would be below the ${ }^{3} \mathrm{He}+{ }^{3} \mathrm{He}$ threshold $(11.5 \mathrm{MeV})$. However, before we can draw any conclusion about this resonance we would like in a near future to improve this experiment by adding more statistic to these data and also by measuring this reaction at some other backward angles.

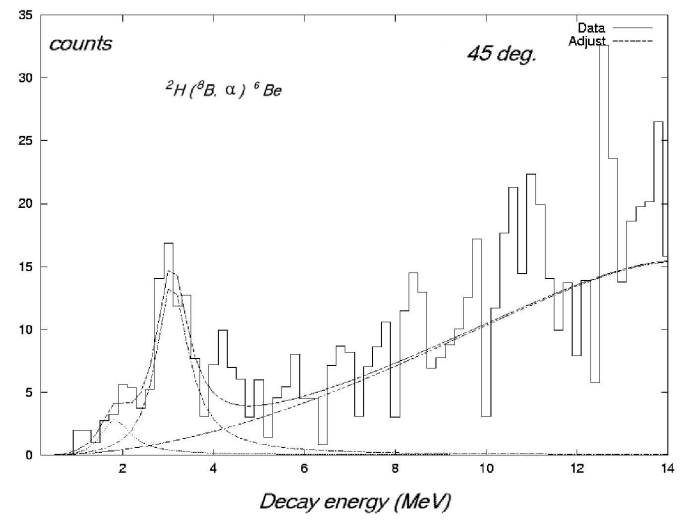

Figure 2. Alpha spectrum from the ${ }^{2} \mathrm{H}\left({ }^{8} \mathrm{~B}, \alpha\right){ }^{6} \mathrm{Be}$ reaction. Energy is measured from the ${ }^{4} \mathrm{He}+2 \mathrm{p}$ decay threshold of ${ }^{6} \mathrm{Be}$. The solid line is a preliminary lineshape of a fit with a Breit-Wigner function considering only the ground-state and the first excitated state in ${ }^{6} \mathrm{Be}$. The background is a 4-body phase-space background.

We intend to continue the search for resonances in these light nuclei by using the installed radioactive ion beam facility in Brazil (RIBRAS). For instance, by using this system to produce ${ }^{7} \mathrm{Be}$ radioactive beam we can performe the ${ }^{3} \mathrm{He}\left({ }^{7} \mathrm{Be}, \alpha\right){ }^{6} \mathrm{Be}$ reaction. A similar reaction, ${ }^{3} \mathrm{He}\left({ }^{7} \mathrm{Li}, \alpha\right){ }^{6} \mathrm{Li}$, with stable ${ }^{7} \mathrm{Li}$ beam from the Pelletron Laboratory of the São Paulo University, has been shown to be a very good reaction to populate resonances in ${ }^{6} \mathrm{Li}[22]$.

\section{The Project RIBRAS}

We intend to continue the spectroscopic investigation of exotic nuclei and the investigation of some nuclear reaction of astrophysical interest by using low energy radioative ion beams reactions. The Direct Nuclear Reaction and Exotic Nuclei group in the Pelletron Laboratory are working in the installation of a double solenoid system to produce secondary low energy radioactive ion beams at the PelletronLINAC laboratory in University of Sao Paulo, Brazil.

The instalattion of such double superconducting solenoid system at Pelletron-LINAC laboratory is almost finished now and will be tested in early 2004. This system was conceived based in the Notre Dame-Michigan Twinsol facility but with higher field integral. Both solenoids were bench tested successfuly on April, 2002. In a first phase the primary beam from the Pelletron Tandem will allow to produce basically the same secondary beams as at the Notre Dame Twinsol facily, i.e., ${ }^{6} \mathrm{He},{ }^{7} \mathrm{Be},{ }^{8} \mathrm{Li}$ and ${ }^{8} \mathrm{~B}$ with the intensity of about $10^{4}$ to $10^{5}$ particle per second. However, the proposed RIBRAS facility, though it uses essentially the same components as the Notre Dame-Michigan Twinsol facility, it will have several important advantages provided by the linear post-accelerator (LINAC). In particular, higher-energy (up to $10 \mathrm{MeV} /$ nucleon), higher mass $(\mathrm{A}=80)$ radioactive ion beams can be produced with beam purities approaching $80 \%$ in many cases. Higher purity may be achieved by using differential energy loss in an energy degrader foil, which might be located at the crossover point between the magnets, shifting the beam contaminants out of the bandpass of the second solenoid. A more detailed description of this system can be found in ref. [23]. The system shall be in operation by early 2004 using the primary beam from the $8 \mathrm{MV}$ Pelletron Tandem. The large angle acceptance and the multiconfiguration and versatility of this system will allow many interesting experiments with low energy radioactive ion beams to be performed.

\section{References}

[1] P.G. Hansen, A.S. Jensen, and B. Jonson, Annu. Rev. Nucl. Part. Sci. 45, 591 (1995).

[2] A. Navin, et al., Phys. Rev. Lett 81, 5089 (1998).

[3] T. Aumann, et al., Phys. Rev. Lett. (in press).

[4] V. Maddalena, et al. (to be published).

[5] V. Guimarães, et al., Phys. Rev. C 61064609 (2000).

[6] C. E. Rolfs and W. S. Rodney, Caudrons in the Cosmos, Nuclear Astrophysics, Ed. University of Chicago, 1988.

[7] D. Galli, et al., Nucl. Phys. A 688, (2001) p. 530c.

[8] B. M. Sherrill, D. J. Morrissey, J. A. Nolen Jr, and J. A. Winger, Nucl. Instrum. Methods Phys. Res. B56 \& 57, 1106 (1991).

[9] H. Scheit et al., Nucl. Instr. Meth. A422, 124 (1999).

[10] B. M. Sherrill (to be published); J. Yurkon et al., Nucl. Inst. Meth. A (1999) (to be published).

[11] GEANT, CERN Library Long Writeup W5013 (1994).

[12] F. Ajzenberg-Selove, Nucl. Phys. A523, 1 (1991).

[13] B. A. Brown, private comunication, and E. K. Warburton and B. A. Brown, Phys. Rev. C 46, 923 (1992).

[14] J. A. Tostevin, J. Phys. G: Nucl. Part. Phys. 25735 (1999).

[15] F. C. Barker, Aust. J. Phys. 33, 177 (1980).

[16] A. E. L. Dieperink and T. de Forest, Jr., Phys. Rev. C 101787 (1996).

[17] A. Azhari, V. Burjan, F. Carstoiu, H. Dejbakhsh, C. A. Gagliardi, V. Kroha, A. Mukhamedzhanov, L. Trache, and R. E. Tribble, Phys. Rev. Lett 82 (1999) 3960.

[18] N. Imai, N. Aoi, S. Kubono et al., Nucl. Phys. A688, 281c (2001). 
[19] L. Trache et al., Phys. Rev. Lett. 87, 271102 (2001).

[20] V. Guimarães, R. Kuramoto, R. Lichtenthäler, G. Amadio, E. Benjamim, P. N. de Faria, A. Lépine-Szily, G. F. Lima, J. J. Kolata, G. Rogachev, F. D. Becchetti, T. O'Donnell, Y. Chen, J. Hinnefeld, and J. Lapton, Nucl. Phys. A722, 341-346c (2003).

[21] M. Y. Lee, F.D. Becchetti, T. W. O’Donnel, D. A. Roberts, J. A. Zimmerman, V. Guimarães, J. J. Kolata,, D. Peterson,
P. Santi, P. A. DeYoung, G. F. Peaslee, and J. D. Hinnenfeld. Nucl. Instr. and Meth. in Phys. Res. A 422, (1999) p.536.

[22] R. Lichtenthäler, et al. to be published.

[23] R. Lichtenthäler, A. Lépine-Szily, V. Guimarães, G. F. Lima, and M. S. Hussein Nucl. Instrum. and Methods in Phys. Res. Abf 505, 612c (2003). 